\title{
ESTUDO RETROSPECTIVO DE LATRODECTISMO NA BAHIA, BRASIL
}

\author{
Rejâne Maria Lira-da-Silva, Graciela Brige Matos, \\ Roney Orismar Sampaio e Tania Brazil Nunes
}

\begin{abstract}
O trabalho apresenta um estudo retrospectivo de setenta e sete casos de latrodectismo no Estado da Babia, Brasil, de agosto de 1980 a julho de 1990. Os dados foram levantados nos livros de registro e arquivo de fichas do CIAVE. O agente etiológico em $28 \%$ dos acidentes aracnídeos foi a espécie $\mathrm{L}$. curacaviensis e a maior incidência foi registrada no meio urbano (57\%), em individuos do sexo masculino (70\%) e faixa etâria de 10 a 29 anos (58\%). Os principais sinais locais foram dor (56\%), pápula eritematosa (21\%) e edema discreto (17\%), e os sistêmicos foram dor em membros inferiores (29\%), tremores e contraturas (29\%), sudorese (28\%) parestesia em membros (21\%) e dor abdominal (17\%). O tratamento foi sintomático em $67 \%$ dos casos $e$ específico em 21\%. O tempo de permanência bospitalar após o uso do soro antilatrodectus foi menor que 24 boras em $64 \%$ dos casos.
\end{abstract}

Palavras-chaves: Latrodectismo. Latrodectus curacaviensis. Estado da Babia.

Designa-se com o nome latrodectismo os acidentes causados no homem pela picada das aranhas do gênero Latrodectus (Walckenaer,1805) com quadro sintomático tão típico e definido que permite considerá-los como uma entidade clínica ${ }^{18}$.

O gênero Latrodectus distribui-se amplamente nas zonas tropicais e subtropicais do globo. Somente três espécies deste gênero ocorrem no Brasil, L. geometricus (Koch, 1841), L. mactans (Müller, 1805) e $L$. curacaviensis (Müller, 1776). Esta última, ocorre desde o Canadá até a Patagônia e no Brasil, ao longo da costaí. No Estado da Bahia é encontrada nas regiões norte, centronorte e sul' ${ }^{15}$. No entanto, esta distribuição provavelmente é muito mais ampla, já que faltam dados de outras localidades do Estado.

A ocorrência do latrodectismo tem sido relatada na maioria dos países tropicais e subtropicais, desde a antiguidade 246101112131617

\footnotetext{
Laboratório de Animais Peçonhentos, Departamento de Zoologia, Instituto de Biologia, Universidade Federal da Bahia e Centro de Informações Antiveneno da Bahia (CIAVE) Sanvador, BA.

Endereço para correspondência: Prof ${ }^{\mathrm{a}}$. Rejâne Maria Lira-daSilva. Laboratório de Animais Peçonhentos, Dept ${ }^{\circ}$ de Zoologia, Instituto de Biologia, Universidade Federal da Bahia, Campus Universitário de Ondina, 40170-210, Salvador, Bahia, Brasil.

Tel: (071) 247-3744; Fax: (071) 245-6909.

Recebido para publicação em 25/07/94.
}

1920. O primeiro relato de acidentes por viúvanegra no Brasil foi feito em 1948 por Machado ${ }^{8}$ no estado do Rio de Janeiro, sem o registro do agente etiológico. Posteriormente, foram assinalados casos, na década de 60 nas cidades do Rio de Janeiro e Niterói, e mais recentemente (1990), na cidade de Agudos (SP) ${ }^{3}$. Na Bahia, em 1985 Rodrigues \& Nunes ${ }^{16}$ apresentaram o primeiro caso comprovado de latrodectismo no País e em 1988, Araújo \& Souza ${ }^{1}$ realizaram um estudo clínico comparativo dos acidentes.

O objetivo deste trabalho foi demonstrar a ocorrência de acidentes por Latradectus curacaviensis no Estado da Bahia, Brasil no período de agosto de 1980 a julho de 1990 e estabelecer as diferenças entre os casos tratados com sintomáticos e os tratados com a soroterapia específica, no que diz respeito à permanência hospitalar.

\section{MATERIAL E MÉTODOS}

Os dados dos casos de latrodectismo registrados no Estado da Bahia, de agosto/1980 a julho/1990, foram levantados nos livros de registro do Centro de Informações Antiveneno da Bahia (CIAVE) e, posteriormente, revisados no seu arquivo de fichas. De setenta e sete casos (77), foram localizadas cinqüienta e duas (52) fichas em arquivo, das quais obtiveram-se os dados de observação e evolução clínica, preenchidas na emergência do Hospital Central Roberto Santos 
Lira-da-Silva RM, Matos GB, Sampaio RO, Nunes TB. Estudo retrospectivo de latrodectismo na Babia, Brasil. Revista da Sociedade Brasileira de Medicina Tropical 28:205-210, jul-set, 1995.

(HCRS) onde os pacientes foram assistidos. Para o caso clínico descrito foi utilizado também o prontuário médico do HCRS.

As informações que constam do livro de registro são: data de solicitação de atendimento ao CIAVE/BA, local do acidente, idade e sexo dos pacientes e uso do soro.

As aranhas causadoras dos acidentes referidas neste trabalho foram identificadas pelo Laboratório de Animais Peçonhentos da Universidade Federal da Bahia (LAP/UFBA).

O soro antilatrodectus (SALAT) utilizado no tratamento dos pacientes foi proveniente do Instituto Malbran (Argentina).

Foram considerados casos prováveis aqueles cujos agentes etiológicos não foram identificados, embora apresentassem sintomas compativeis com o latrodectismo.

\section{RESULTADOS}

O latrodectismo foi identificado em $28 \%$ (77) dos casos araneídicos (277), dos quais $21 \%$ foram comprovados pelo agente etiológico, Latrodectus curacaviensis (Figura 1).

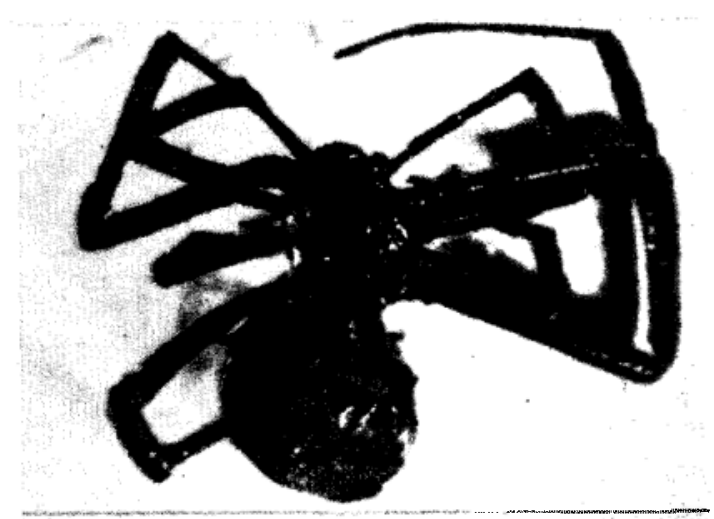

Figura 1 - Fêmea de Latrodectus curacaviensis.Agente etiológico do caso clínico descrito.

A distribuição geográfica de latrodectismo na Bahia demonstra maior incidência nas regiões Metropolitana de Salvador, CentroNorte e Nordeste (Figura 2).

A maior freqüência de acidentes foi registrada na área urbana (57\%), em ambientes peridomiciliares, e em pacientes que estavam dormindo dentro de suas casas. Os meses de dezembro, fevereiro, março, abril e maio apresentaram a maior casuística (Figura 3), observando-se uma diferença marcante, entre os casos do sexo masculino (70\%) e feminino (30\%). O maior número de casos ficou concentrado na faixa etária entre 10 e 29 anos (58\%) sem registro de acidentes com crianças abaixo de um ano.

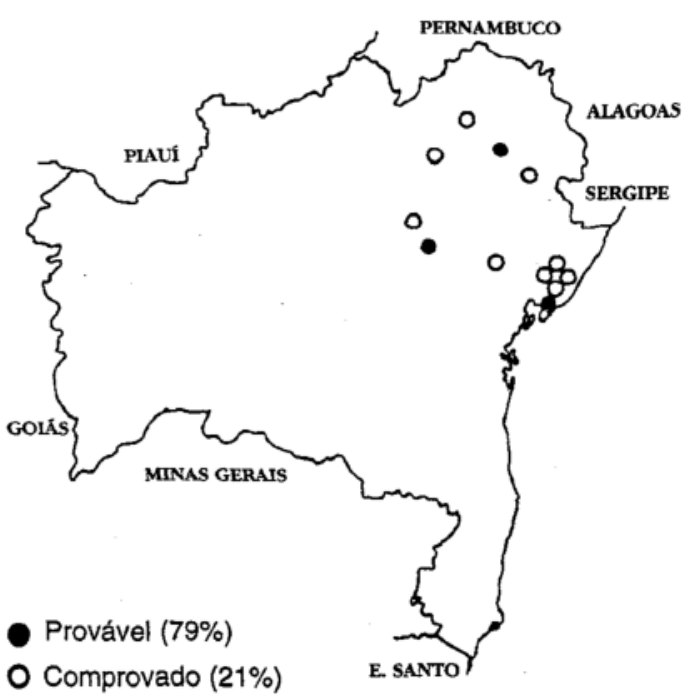

Figura 2 - Distributçào geográfica de latrodectismo na Bahia.

Os principais sinais locais observados foram: dor $(56 \%)$, pápula eritematosa $(21 \%)$, edema discreto (17\%) e sudorese (13\%). Nenhum dos pacientes apresentou quadro local de maior magnitude ao exame clínico. Os sistêmicos caracterizaram-se por: dor em membros inferiores (MMII) (29\%), tremores e contraturas (29\%), sudorese generalizada (28\%), parestesia em membros superiores (MMSS) e/ou MMII (21\%) e dor abdominal (17\%) (Tabela 1). Não foi registrado óbito no período.

O tratamento utilizado foi sintomático em $67 \%$ dos pacientes e em $21 \%$ foi o soro antilatrodectus (SALAT) (Figura 4). O tempo de permanência hospitalar após o uso do SALAT foi menor que 24 horas em $64 \%$ dos casos.

\section{Descrição de um caso clínico por Latrodectus curacaviensis}

E.N.R.S., sexo masculino, 6 anos, procedente de São Cristovão, Salvador, Bahia, 
Lira-da-Silva RM, Matos GB, Sampaio RO, Nunes TB. Estudo retrospectivo de latrodectismo na Babia, Brasil. Revista da Sociedade Brasileira de Medicina Tropical 28:205-210, jul-set, 1995.

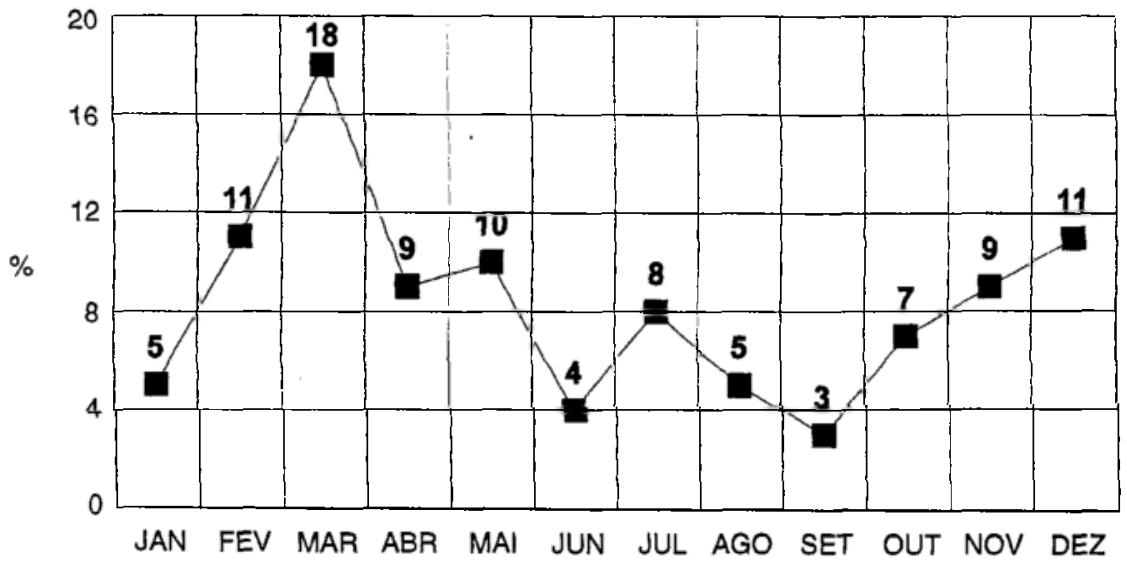

Fonte: CIAVE-SERVS-SESAB

Figura 3 - Freqüência mensal de acidentes por Latrodectus na Babia (1980-1990).

deu entrada em 28/04/90 na emergência do HCRS às 12:55h, com história de ter sido picado há cerca de 1 hora por aranha de nome vulgar viúva-negra, que trouxe consigo, sendo identificada como Latrodectus curacaviensis (Figura 1). Ao exame, o paciente referiu dor local $e$ abdominal de forte intensidade, mostrou-se agitado, com períodos de sonolência, gemente, em regular estado geral, afebril, taquipnéico, corado, anictérico, freqüência cardíaca de $120 \mathrm{bpm}$ e freqüência respiratória de $28 \mathrm{ipm}$. O abdome apresentavase rígido, em tâbua e extremidades sem

\section{a}

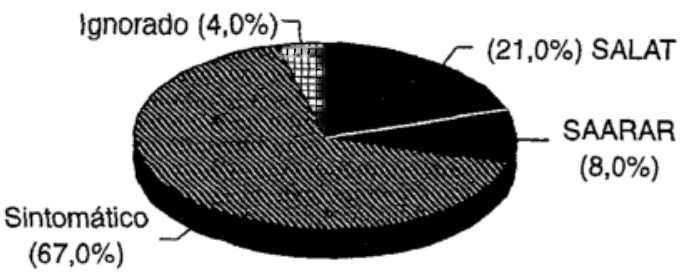

Legenda: SALAT = soro antilatrodectus; SAARAR $=$ soro antiaracnídico

Figura 4 - Tratamento utilizado em 77 pacientes picados por Latrodectus na Babia (1980-1990). alterações. À ausculta do aparelho respiratório, observou-se crepitação discreta em bases. $O$ paciente foi medicado com baralgim ${ }^{\mathrm{L}}(0,6 \mathrm{ml}$, via oral), diazepan ( $5 \mathrm{ml}$ endovenoso diluído), fenergam ${ }^{\circledR}$ (meia ampola, endovenoso) e

Tabela 1 - Principats stnats e sintomas observados em 77 fichas médjcas, de acidentes por Latrodectus na Babia, no perído de ago 1980 a jul 1990.

\begin{tabular}{|c|c|c|}
\hline Sinais e Sintomas & $\mathrm{N}^{2}$ & $\%$ \\
\hline Dor local & 43 & 56,0 \\
\hline Dor em MMII & 22 & 29,0 \\
\hline Tremores e contraturas & $2^{+}$ & 29,0 \\
\hline Sudorese generalizada & 20 & 28,0 \\
\hline Pápula eritematosa local & 16 & 21,0 \\
\hline Parestesia em MMII e/ou MMSS & 16 & 21,0 \\
\hline Dor abdominal & 13 & 17,0 \\
\hline Edema local discreto & 13 & 17,0 \\
\hline Agitação & 11 & 14,0 \\
\hline Ansiedade & 10 & 13,0 \\
\hline Sudorese local & 10 & 13,0 \\
\hline Dificuldade de deambular & 9 & 12,0 \\
\hline Dor em MMSS & 9 & 12,0 \\
\hline Hipertermia & 9 & 12,0 \\
\hline Dores generalizadas & 8 & 10,0 \\
\hline Dor precordial & 8 & 10,0 \\
\hline Formigamento local & 8 & 10,0 \\
\hline Sonolência & 8 & 10,0 \\
\hline Cefaléia & 6 & 8,0 \\
\hline Dores articulares & 6 & 8,0 \\
\hline Tontura & 6 & 8,0 \\
\hline Dispnéia leve & 5 & 6,0 \\
\hline Fácies latrodectísmica & 5 & 6,0 \\
\hline Oligúria & 5 & 6,0 \\
\hline Taquicardia & 5 & 6,0 \\
\hline Bradicardia & 4 & 5,0 \\
\hline Lesão punctiforme local & 4 & 5,0 \\
\hline Vômitos & 4 & 5,0 \\
\hline Hipereflexia & 3 & 4,0 \\
\hline Hipertensão arterial & 3 & 4,0 \\
\hline Outros* & 39 & 51,0 \\
\hline
\end{tabular}

MMII (membros inferiores)/MMSS (membros superiores).

* Nesta categoria estão agrupados os sinais e sintomas com percentual abaixo de $4,0 \%$.

Fonte: CIAVE - SERVS - SESAB. 
Lira-da-Silva RM, Matos GB, Sampaio RO, Nunes TB. Estudo retrospectivo de latrodectismo na Babia, Brasil. Revista da Sociedade Brasileira de Medicina Tropical 28:205-210, jul-set, 1995.

gluconato de cálcio a 10\% (2 ampolas, endovenoso, lentamente).

Segundo avaliaçâo clínica posterior (no mesmo dia), o paciente apresentou edema bipalpebral, temperatura de $37,8^{\circ} \mathrm{C}$ e hipertonia generalizada. Continuou em uso do fenergam $B)(1 / 2$ ampola, intramuscular), gluconato de cálcio a $10 \%$ ( $4 \mathrm{ml}$ diluído em $100 \mathrm{ml}$ de solução glicosada a $5 \%$ endovenoso) e diazepan $(5 \mathrm{mg}$ diluído em $10 \mathrm{ml}$ de solução glicosada a 5\%, endovenoso a cada 8 horas), este último foi suspenso sem que a ficha do paciente indicasse a data da suspensão.

Em 29/04/90, às 10:30h, apresentava-se sonolento, pulso de $136 \mathrm{bpm}$, temperatura de $37,8^{\circ} \mathrm{C}$, abdome rígido e doloroso à palpação. Às 14:50h, referiu fraqueza, cansaço e melhora das contraturas. $\grave{A}$ noite, a temperatura manteve-se em $37,8^{\circ} \mathrm{C}$ e foi administrado antitérmico.

Em 30/04/90, o paciente queixou-se de dores em MMII, principalmente nos pés, apresentava "fácies latrodectísmica", abdome contraído, priapismo e freqüência cardíaca de $92 \mathrm{bpm}$.

Em 01/05/90, fez uso do SALAT (trazido do Instituto Malbran, Argentina) por volta das 19:00h. Em 02/05/90 às 8:50h apresentou febre, diarréia e dor abdominal (tipo cólica); às 11:30h estava em bom estado geral e às 17:00h recebeu alta hospitalar.

\section{DISCUSSÃO}

Na região Metropolitana de Salvador, $L$. curacaviensis ocorre em cavidades de barrancos, embaixo de cascas de coco, de pedras, entre as folhas de plantas herbáceas, nos jardins ou no interior de residências?, divergindo das observações de Machado $^{8}$ e Bucherl $^{4}$, que afirmam que estas aranhas são encontradas em plantas na restinga das praias do Rio de Janeiro e Niterói.

A maior percentagem dos acidentes procederam da Capital (57\%), especialmente de áreas urbanizadas e o histórico dos casos estudados, evidenciou o hábito urbano e peridomiciliar dessa espécie, favorecendo a ocorrência de acidentes, fato semelhante ao observado para $L$. mactans na América do Norte $^{10}$.

A freqüência máxima de acidentes ocorreu nos meses mais quentes (dezembro a maio) (Figura 3) correspondendo ao mesmo período de latrodectismo na Argentina ${ }^{71}$ e no Chile ${ }^{19}$.
Segundo Maretic ${ }^{11}$ a incidência dos acidentes quanto ao sexo depende do hábito da aranha (rural ou doméstico) e do local de trabalho da vítima. Realmente, a maior frequência de acidentes em pacientes do sexo masculino (70\%) e dentro da faixa etária 10-29 anos (58\%) sugere uma relação com a atividade exercida pelo acidentado (jardinagem, capinagem), embora Araújo \& Souza $^{1}$ não encontrassem para essa mesma região (Bahia) diferenças significativas quanto ao sexo, possivelmente devido ao número de casos estudados (cerca de 30). Relação semelhante foi descrita para países do Mediterrâneo ${ }^{1011}{ }^{13}$, Argentina ${ }^{714}$ e Chile ${ }^{19}$, onde o latrodectismo ocorre com predominância em campos cultivados, com $70 \%$ a $80 \%$ dos acidentes atingindo o sexo masculino, mais exposto por trabalhos rurais (devido a precária mecanização da agricultura).

Os sinais locais caracterizaram-se por dor (56\%), pápula eritematosa (21\%), edema discreto (17\%) e sudorese local (13\%), já referidos por diversos autores ${ }^{14}>1415$ para acidentes por Latrodectus sul-americanas. Essa semelhança é acentuada pela ausência de reações locais como área pálida demarcada por bordas avermelhadas/azuladas, bolhas, edema, necrose cutânea e/ou equimose, encontradas nos acidentes por Latrodectus européias ${ }^{12} 13$.

Dos sintomas que caracterizam o quadro sistêmico, tanto os simpático-tônicos quanto os vago-tônicos ${ }^{14}$, podem ser evidenciados pelo quadro álgico (>56\%), tremores e contraturas (29\%) e sudorese generalizada (28\%) (Tabela 1).

A ausência de casos fatais constatada até hoje no Brasil, coincide com as observações de Maretic $^{12}$ e Grisolia e cols ${ }^{7}$ nas regiões do Mediterrâneo (incluindo o Sul da Rússia, Israel e Africa do Norte) e Argentina, respectivamente. Casos fatais ocorreram no Chile $(2,4 \%)^{19}$, Itália, Iugoslávia, e U.S.A. ( 4 a $6 \%)^{12}$. Estes registros reforçam a sugestão de Maretic $^{12}$, que o modo de ação das toxinas de Latrodectus (refletidas nas manifestações clínicas) embora semelhante, varia em gravidade de acordo com as espécies.

O tratamento específico - uso do SALAT (soro antilatrodectus) - está diretamente relacionado à rápida melhora do quadro inicial e alta hospitalar. Martino e cols ${ }^{14}$ referem que o tratamento com soro específico demonstra 
Lira-da-Silva RM, Matos GB, Sampaio RO, Nunes TB. Estudo retrospectivo de latrodectismo na Babia, Brasil. Revista da Sociedade Brasileira de Medicina Tropical 28:205-210, jul-set, 1995.

melhora dentro das primeiras três horas após sua administração; no entanto, a dificuldade na obtenção do soro obriga a busca de novos agentes terapêuticos de ação rápida e eficaz, como a administração de sais de cálcio, clorpromazina, prostigmina, hidratação, cardioestimulantes, analgésicos, etc. Segundo Maretic $^{12}$, o tratamento que mais tem demonstrado sucesso é o que utiliza o cálcio e o SALAT, especialmente a administração conjunta de ambos. Grisolia e cols ${ }^{7}$ referem que $100 \%$ dos pacientes tratados com o SALAT, manifestou diminuição da sintomatologia uma hora após a sua aplicação, e recuperação quase total dentro de três horas. Em nossa casuistica, dos $21 \%$ (11) dos pacientes que fizeram uso do SALAT, $64 \%$ teve alta hospitalar em menos de 24 horas, concordando com os dados obtidos por Araújo \& Souza ${ }^{1}$ na mesma região. Os casos tratados apenas com sintomáticos, evoluíram mais lentamente; apesar disso, Bucharetchi ${ }^{3}$ refere que existem divergências quanto à indicação de soroterapia, e que alguns autores têm recomendado sua aplicação somente em casos com intenso envolvimento sistêmico.

Consideramos que o critério tempo de permanência hospitalar total não é um bom parâmetro para avaliar a evolução dos casos tratados com soro específico, pois não sendo disponível no Brasil, a permanência prolongada do paciente no hospital decorre muitas vezes da demora do serviço em obter o soro de outros países.

\section{Conclusão}

Os dados demonstram até agora a Bahia como responsável pela maior casuística de acidentes por viúva-negra no Brasil, 77 casos no período de 1980 a 1990.

$\mathrm{O}$ agente etiológico do Latrodectismo na Bahia é a aranha Latrodectus curacaviensis, encontrada freqüentemente na região urbanizada de Salvador e responsável pela maior freqüência de acidentes araneídicos (28\%), no período de agosto de 1980 a julho de 1990. O período de maior freqüência dos acidentes compreende os meses de dezembro a maio.

O envenenamento produzido por esta aranha causa quadro clínico semelhante aos descritos na literatura, porém de leve a média gravidade, sem ocorrência de óbitos.
O número de acidentes registrados na região indica a necessidade imediata de disponibilidade do soro específico - SALAT no Brasil, pois todos os autores concordam que os pacientes adequadamente tratados se recuperam em um período menor que 24 horas.

\section{SUMMARY}

This work is a retrospective study of latrodectism in the State of Babia, Brazil, from August 1980 to July 1990. The data concerning the accidents were obtained from file cards at the Antivenom Information Center of Babia (AVTCB). Latrodectus curacavienis was the etbiologic agent identified in $28 \%$ of the arachnid accidents. The major incidence was registered in urban area (57\%) affecting men (70\%) more than women, with 10 to 29 year-old age group (58\%). Local pain (56\%), erytbematous papula (29\%) and light oedema (17\%) were the principal local symptoms. Pain in the limbs (29\%), tremor and rigidities (29\%), sweating (28\%), limbs and arms paresthesia (21\%) and abdominal pain (17\%) were systemic ones. The treatment was mainly symptomatic (67\%) and antivenin serum was used in 21\% of the cases. After serotherapy, 64\% of the patients left the bospital witbin less than 24 bours.

Key-words: Latrodectism. Latrodectus curacaviensis. Babia State.

\section{AGRADECIMENTOS}

Os autores agradecem a valiosa colaboração do Dr. Daniel Santos Rebouças e dos plantonistas do CIAVE pela coleta dos dados retrospectivos; À Fundação Banco do Brasil pelo suporte financeiro e a Fernando Daltro Júnior pelas fotos.

\section{REFERÊNCIAS BIBLIOGRÁFICAS}

1. Araújo CL, Souza IM. Estudo clínico e comparativo do latrodectismo na Bahia. Revista da Sociedade Brasileira de Toxicologia 1:53-55, 1988.

2. Brazil V, Vellard J. Contribuition a l'etude du venin des araignées. Memórias do Instituto Butantan, Tomo II, Fasc. único, 1925.

3. Bucharetchi F. Acidentes por Latrodectus. Latrodectismo. In: Schvartsman $\mathrm{S}$ (ed) Plantas venenosas e animais peçonhentos. $2^{a}$ edição, Editora Sarvier p. 204-206, 1992.

4. Bücherl W. Invertebrados. As Aranhas. Coleção Cientistas do Amanhã. Edart Livraria e Editora. São Paulo, 1972. 
Lira-da-Silva RM, Matos GB, Sampaio RO, Nunes TB. Estudo retrospectivo de latrodectismo na Babia, Brasil. Revista da Sociedade Brasileira de Medicina Tropical 28:205-210, jul-set, 1995.

5. De Biasi P. Variações em aranhas do complexo Latrodectus mactans - Latrodectus curacaviensis (Araneae; Theridiidae). Revista Brasileira de Biologia 30:233-244, 1970.

6. Gajardo-Tobar R. El araneismo en el mundo tropical y sub-tropical. Memórias do Instituto Butantan 33:454-54, 1966.

7. Grisolia GS, Peluso FO, Stanchi NO, Francini F. Epidemiologia del latrodectismo en la província de Buenos Aires, Argentina. Revista de Saúde Pública 26: 1-5, 1992.

8. Machado O. Latrodectus mactans sua ocorrência no Brasil. Boletim do Instituto Vital Brazil 5:153160,1948

9. Maciel TR, Silva RML, Silva LLC. Estudo do comportamento de Latrodectus curacaviensis (Araneidae: Theridiidae). In: Resumos do XV Congresso Brasileiro de Zoologia, Curitiba p. 633, 1988.

10. Maretic Z. Latrodectism in mediterran coutries, including South Russia, Israel, and North Africa. In: Bücherl W, Buckley EE, Deulofeu V (eds) Venomous animals and their venoms. Academic Press, Nova Yorque, Vol. III, p. 299-309, 1971.

11. Maretic Z. Venoms of Theridiidae, Genus Latrodectus. Epidemiology of envenomation, symptomatology, pathology and treatment. In: Bettini S (ed) Handbook of experimental pharmacology. Arthropds venoms. SpringerVerlag Berlin/Heideberg, Vol. 48, p.185-212,1978.

12. Maretic Z. Latrodectism: variations in clinical manifestations provoked by Latrodectus species of spiders. Toxicon 21:457-466, 1983.
13. Maretic Z, Gonzalez-Lourenzo D. Caráter professional del Latrodectismo en Paises Mediterrâneos, com especial referência a experiência en Iugoslávia y España. Revista Clinica Española Tomo 160: 225-228, 1981.

14. Martino OA, Mathet $\mathrm{H}$, Masini $\mathrm{RD}$, Grasso AI, Thompson RM, Gondel C, Bosch JE. Emponzoñamiento humano provocado por venenos de origem animal. Estudo epidemiológico, clínico y experimental. Sector Educación para la Salud. Argentina, 1979.

15. Projeto Estudo dos Animais Peçonhentos da Bahia. Relatório Final. Departamento de Zoologia, Instituto de Biologia da Universidade Federal da Bahia. Salvador: Laboratório de Animais Peçonhentos, Dep. de Zoologia, Instituto de Biologia, Universidade Federal da Bahia, vol II, 1992.

16. Rodrigues DS, Nunes TB. Latrodectismo na Bahia. Revista Baiana de Saúde Pública. 12:38-43, 1985.

17. Rodrigues JH, Peres MSZ, Nóbrega MM,Torres, JB. Acidentes com aracnideos. Revista HCPA, 6:9196, dez, 1986.

18. Sampayo R. Latrodectus mactans $y$ Latrodectismo. Tese de doutorado, Buenos Aires, Argentina, 1942.

19. Schenone H. Latrodectismo y loxoscelismo en Chile. Incidência, características clínicas, prognóstico, tratamento y prevencion. Memórias do Instituto Butantan 33:207-211, 1966.

20. Vellard J. El araneísmo en Bolivia. Memórias do Instituto Butantan 33: 699-700, 1966. 